\title{
POTENCIAIS INTERAÇÕES MEDICAMENTOSAS EM PRESCRIÇÕES DO HOSPITAL MUNICIPAL DA CIDADE DE CUITÉ - PARAÍBA
}

\author{
Jéssica Karinny Dantas de Oliveira ${ }^{1}$, Marina de Souza Farias Santos ${ }^{1}$, Karoline \\ Gomes Dias Bezerra², Fernando de Sousa Oliveira ${ }^{3}$ \\ ${ }^{1}$ Farmacêutica pela Universidade Federal de Campina Grande, Cuité-PB, Brasil. \\ ${ }^{2}$ Graduanda em Farmácia da Universidade Federal de Campina Grande, Cuité-PB, Brasil. \\ ${ }^{3}$ Professor do Centro de Educação e Saúde, Universidade Federal de Campina Grande, Cuité, \\ PB, Brasil. \\ Email para correspondência: fernandoufcg@hotmail.com
}

\begin{abstract}
Resumo
A avaliação de interações farmacológicas em prescrições hospitalares de medicamentos constitui um importante meio de garantir uma efetiva farmacoterapia, evitando danos e garantindo o tratamento adequado ao paciente. Objetivou-se avaliar as potenciais interações medicamentosas em prescrições de pacientes do Hospital Municipal de Cuité/PB. Realizou-se um estudo transversal, descritivo, quantitativo e qualitativo, no período de abril a agosto de 2017, a partir de prescrições de medicamentos. Os dados foram coletados por meio de um formulário específico e as potenciais interações foram identificadas utilizando o Drug-Reax System do software Micromedex ${ }^{\circledR}$ Health Series. Dentre as 325 prescrições avaliadas, 120 apresentaram alguma potencial interação, representando uma alta prevalência, principalmente, em pacientes acima de 60 anos. Observou-se uma média de 3,6 fármacos por prescrição com 0,7 de interação. A maioria dos pacientes que utilizou entre 6 e 10 fármacos apresentou algum tipo de potencial interação. O fármaco presente no maior número de prescrições e mais envolvido em possíveis interações foi a dipirona. Quanto a classificação, a maioria das interações foi descrita com grau de severidade maior, razoável documentação e início de ação não especificado. Sendo assim, é indispensável à monitorização das prescrições, aperfeiçoando o manejo clínico e evitando futuras complicações.
\end{abstract}

Palavras-chave: Tratamento farmacológico, uso de medicamentos, farmácia.

\begin{abstract}
The evaluation of pharmacological interactions in hospital drug prescriptions is an important means of ensuring effective pharmacotherapy, avoiding damage and ensuring adequate treatment for the patient. The objective was to evaluate the potential drug interactions in prescriptions of patients at the Municipal Hospital of Cuité / PB. A cross-sectional, descriptive, quantitative and qualitative study was carried out from April to August 2017, based on drug prescriptions. Data were collected using a specific form and potential interactions were identified using the
\end{abstract}




\begin{abstract}
Drug-Reax System from the Micromedex® Health Series software. Among the 325 prescriptions evaluated, 120 showed some potential interaction, representing a high prevalence, especially in patients over 60 years of age. There was an average of 3.6 drugs per prescription with 0.7 of interaction. Most patients who used between 6 and 10 drugs had some type of potential interaction. The drug present in the largest number of prescriptions and most involved in possible interactions was dipyrone. Regarding classification, most interactions were described with a greater degree of severity, reasonable documentation and unspecified onset of action. Therefore, it is essential to monitor prescriptions, improving clinical management and avoiding future complications.
\end{abstract}

Keywords: Drug therapy, drug utilization, pharmacy.

\title{
1 Introdução
}

A introdução maciça de novos medicamentos após 1940, trouxe à população a possibilidade de cura de doenças que até então eram fatais, sobretudo no campo das doenças infeciosas. No entanto, os rápidos avanços na pesquisa de novas substâncias ativas e a promoção comercial excessiva dos medicamentos, resultaram na crença da sociedade do poder dos fármacos. A produção em escala industrial, segundo especificações técnicas e legais, fez com que estes produtos alcançassem um papel central na terapêutica, considerado um importante recurso no cuidado ao paciente e quase obrigatórios após a consulta clínica (COSTA; CATARINA; BARATA, 2014).

Assim, com o maior foco na finalidade dos medicamentos, muitas vezes passam despercebidos os males que esses podem ocasionar quando não utilizados corretamente (FORMIGA, 2012).

A associação medicamentosa é entendida como uma prática rotineira em ambientes hospitalares (VIEL et al., 2014), e o uso da polimedicação tem sido útil no tratamento de patologias coexistentes, porém deve-se monitorar essas combinações de fármacos, pois podem acarretar na redução da eficácia e/ou favorecerem o aparecimento de eventos adversos de diferentes gravidades, com a incidência de interações entre fármacos aumentando exponencialmente com o número de medicamentos prescritos (COELHO; BRUM, 2012).

O risco de interação fármaco-fármaco ocorre em $13 \%$ dos pacientes utilizando dois medicamentos e $85 \%$ em pacientes utilizando mais de seis medicamentos (SCRIGNOLI; TEXEIRA; LEAL, 2016). A gravidade potencial da interação é particularmente importante para avaliar o risco/benefício das alternativas terapêuticas. Com dosagem apropriada ou com a modificação do 
regime de administração, os efeitos negativos da maioria das interações podem ser evitados (FERREIRA JR et al., 2016).

Em hospitais, é frequente a relação entre a grande demanda de fármacos prescritos e o risco potencial de interações que ocorrem entre estes, o que vem a ser um problema presente em hospitais públicos e privados (PINTO; SOUZA; CARNEIRO, 2015).

A avaliação da farmacoterapia é um importante meio de analisar a ocorrência de interação medicamentosa e suas implicações clínicas, refletindo a resposta do paciente ao esquema terapêutico utilizado. Com a análise das prescrições, é possível direcionar e adotar planos terapêuticos mais seguros, possibilitando uma melhor assistência e menores danos aos pacientes. O efeito gerado por tais reações pode ser nocivo, se a interação provocar aumento na toxicidade do fármaco. Sendo assim, é de suma importância averiguar e contornar possíveis problemas relacionados ao uso da polifarmácia, reações adversas e a ocorrência de potenciais interações medicamentosas em prescrições a nível hospitalar.

Perante o exposto, o presente estudo teve como objetivo avaliar as potenciais interações do tipo fármaco-fármaco em prescrições de pacientes internados no Hospital Municipal da cidade de Cuité - Paraíba, com intuito de conhecer para melhor monitorar e intervir em prescrições futuras no que se refere a essas interações, melhorando assim a farmacoterapia proposta pelas prescrições hospitalares.

\section{Metodologia}

Trata-se de um estudo transversal, descritivo, com abordagem quantitativa e qualitativa, desenvolvido na sala de arquivos do Hospital Municipal de Cuité/PB, onde ficam arquivadas todas as prescrições que são dispensadas pela farmácia do hospital. A coleta de dados foi procedida por duas estudantes do Curso de Farmácia da Universidade Federal de Campina Grande. A amostra foi constituída por todas as prescrições dos pacientes internados no hospital, durante o período de abril a agosto de 2017, apresentando mais de um medicamento. Apenas a primeira prescrição de cada paciente do hospital durante o período de estudo foi avaliada. 
$\mathrm{Na}$ análise quantitativa foram observadas as seguintes variáveis: média de potenciais interações por prescrição, fármaco mais prescrito, fármaco mais envolvido em interações e prevalência de interações farmacocinéticas e farmacodinâmicas. Os valores foram transformados em porcentagens e os dados foram estruturados empregando-se o programa $E_{x c e}{ }^{\circledR}$.

As potenciais interações encontradas foram classificadas quanto ao mecanismo de ação pelo qual são produzidas (farmacocinético, farmacodinâmico e desconhecido), conforme a severidade (maior, moderada, menor e contraindicada), quanto ao início de ação (rápido, tardio e não especificado) e quanto ao grau de embasamento na literatura (excelente, bom e razoável).

Para análise dos dados, utilizou-se o programa DrugReax System ${ }^{\circledR}$, que faz parte base de dados Micromedex ${ }^{\circledR}$ disponível no Portal de Periódicos Capes.

O estudo foi aprovado pelo Comitê de Ética em Pesquisa do Hospital Universitário Alcides Carneiro da Universidade Federal de Campina Grande sob o parecer № 1.973 .644 e Certificado de Apresentação para Apreciação Ética no 62181416.7.0000.5182.

\section{$3 \quad$ Resultados}

Analisaram-se 325 prescrições durante o período da pesquisa, por meio da base de dados Micromede $x^{\circledR}$, foram encontradas 120 prescrições (37\%) com potencais interações medicamentosas.

Dos 325 pacientes que constituíam a amostra, 176 (54,2\%) eram do sexo feminino, destes, 69 (57,5\%) apresentaram prescrição com algum tipo de potencial interação. Dentre os 149 (45,9\%) pacientes do sexo masculino, 51 $(42,5 \%)$ apresentaram alguma potencial interação medicamentosa nas prescrições (Gráfico 1).

A prevalência de potenciais interações medicamentosas analisadas no estudo está em concordância com os percentuais encontrados em outras pesquisas semelhantes (MOURA; TAVARES; ACURCIO, 2012; ROCHA; MOTA; OLIVEIRA, 2014; MARSILIO; SILVA; BUENO, 2016). Em estudos similares, encontrou-se uma amostra composta majoritariamente por pacientes 
do sexo feminino, sendo as mulheres as que mais procuram atendimento clínico (CEDRAZ; DOS SANTOS JÚNIOR, 2014; SANTOS; FREITAS; COSTA (2016). Apesar de ter sido observada maior frequência de prescrição de fármacos que podem interagir entre si, a variável sexo não foi detectada como fator de risco para a ocorrência de interações medicamentosas (VARALLO; COSTA; MASTROIANNI, 2013).

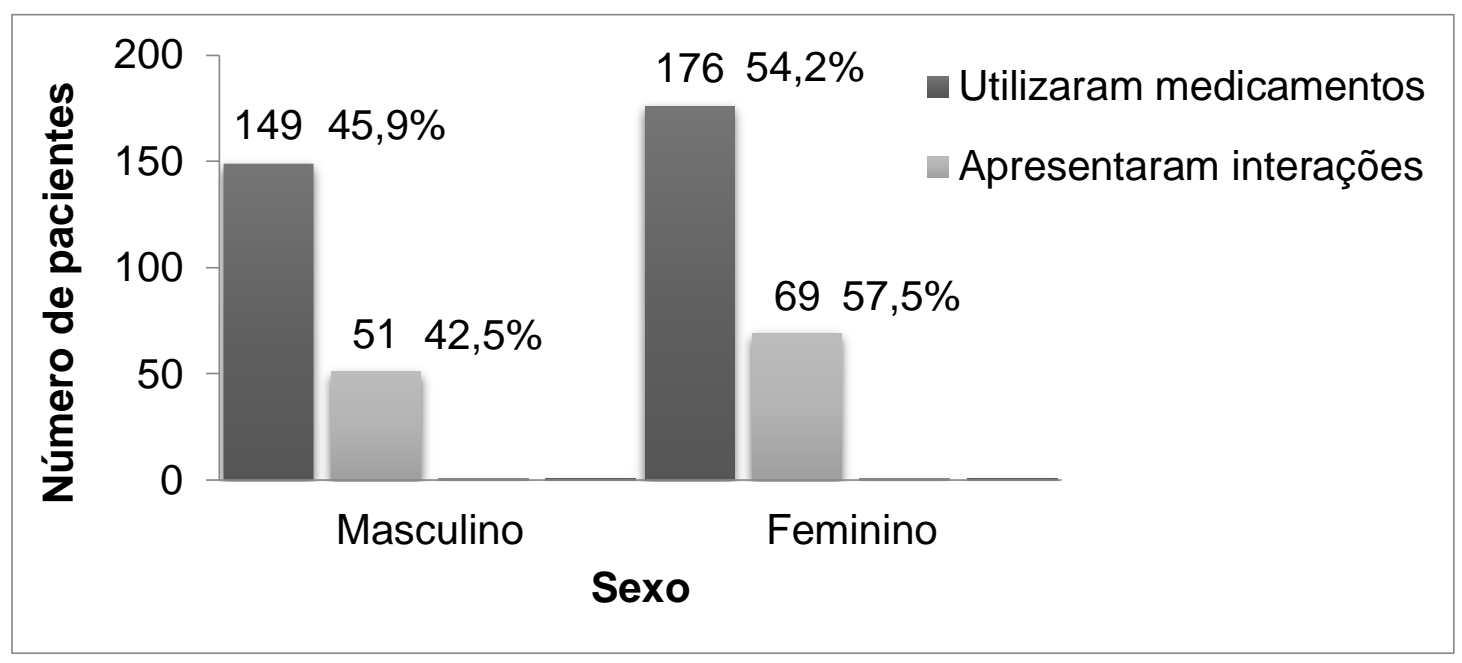

Gráfico 1. Relação entre sexo e número de interações medicamentosas Fonte: elaboração própria.

Dentre as 325 prescrições que constituíram a amostra, 39,1\% eram de pacientes menores de 18 anos, destas 15,8\% apresentaram algum tipo de potencial interação; $17,9 \%$ eram de indivíduos entre 19 e 59 anos de idade, com $23,3 \%$ prescrições expressando algum tipo de potencial interação medicamentosa; $43,1 \%$ das prescrições eram de pacientes que possuíam 60 anos ou mais, com $60,8 \%$ apresentando potenciais interações (Gráfico 2).

Com relação à idade dos pacientes, o resultado encontrado é semelhante a um estudo publicado na literatura, em que a maioria dos pacientes era indivíduos com 60 anos ou mais de idade (SANTOS et al., 2010). A terapia medicamentosa em idosos deve ganhar atenção e cuidado diferenciados, pois mudanças fisiológicas relacionadas ao envelhecimento - por exemplo, modificação da composição corporal, diminuição da produção do suco gástrico, menor quantidade de proteínas plasmáticas, menor teor de água corporal e redução das funções renal e hepática - podem alterar, de forma significativa, a 
farmacocinética e a farmacodinâmica de diversos fármacos. Esse fato, faz com que indivíduos idosos estejam mais suscetíveis a eventos adversos ou terapêuticos mais intensos pelo uso de medicamentos, e por isso necessitam de maior cuidado no âmbito hospitalar (PINTO et al., 2014).

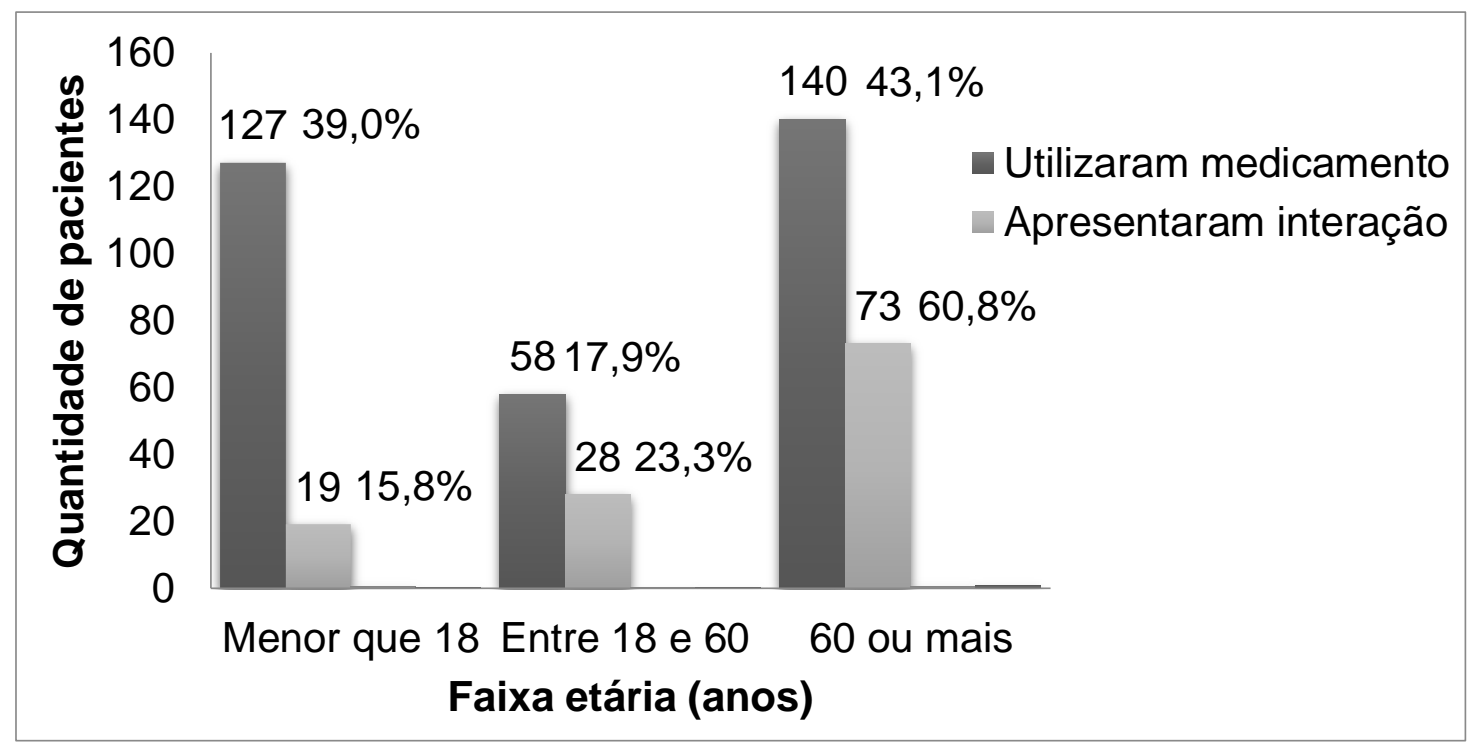

Gráfico 2. Relação entre faixa etária e o número de interações medicamentosas Fonte: elaboração própria.

Identificam-se a presença de 120 prescrições com potenciais interações medicamentosas, no qual totalizou uma média de 0,7 interações por prescrição. Dentre as 214 potenciais interações encontradas nas prescrições, houve uma variedade de 73 interações diferentes. Foi calculada a média de fármacos por prescrição, a qual correspondeu a 3,6 fármacos/prescrição (Tabela 1). O valor de média de fármacos observada está em conformidade com os percentuais encontrados em outros estudos semelhantes (QUEIROZ et al., 2014; ROCHA; MOTA; OLIVEIRA, 2014; LIMA et al., 2016).

Tabela 1. Relação entre fármacos e interações por prescrição

\begin{tabular}{cc}
\hline Características & Valor (média \pm DP) \\
\hline Número de fármacos/prescrição & $3,6 \pm 1,2$ \\
Número de interações/prescrição & $0,7 \pm 0,0$ \\
\hline
\end{tabular}

Fonte: elaboração própria. 
Para as prescrições com até cinco fármacos, um total de $32,6 \%$ das prescrições apresentou alguma potencial interação. As 22 (91,7\%) prescrições com seis a dez fármacos possuía pelo menos uma provável interação (Tabela 2). A quantidade de fármacos prescritos está diretamente relacionada à presença de interações, sendo a polifarmácia responsável pelo surgimento dessas potenciais interações medicamentosas (ROCHA; MOTA; OLIVEIRA, 2014; QUEIROZ et al., 2014; LIMA et al. 2016).

Tabela 2. Relação entre o número de fármacos prescritos e prevalência de potenciais interações medicamentosas

\begin{tabular}{cccc}
\hline $\begin{array}{c}\text { Fármacos } \\
\text { prescritos }\end{array}$ & Prescrições & $\begin{array}{c}\text { Prescrições com } \\
\text { interações }\end{array}$ & $\begin{array}{c}\text { \% Prescrições com } \\
\text { potenciais interações }\end{array}$ \\
\hline Até 5 & 301 & 105 & 32,6 \\
Entre $6-10$ & 24 & 22 & 91,7 \\
\hline \multicolumn{4}{c}{ Fonte: elaboração própria. }
\end{tabular}

Foram encontrados 76 fármacos diferentes prescritos. O fármaco com maior número de prescrições foi a dipirona (analgésico e antitérmico), o qual apresenta-se em 129 (39,7\%) do total de 325 prescrições. Em seguida, o succinato sódico de hidrocortisona (anti-inflamatório corticosteroide) esteve em 120 (36,9\%) prescrições, ceftriaxona (antimicrobiano) em 117 (36,0\%) e o brometo de ipratrópio (anticolinérgico) em 67 (20,6\%), conforme é demonstrado na tabela 3.

No tocante ao fármaco mais prescrito, resultado parecido foi encontrado por um estudo descritivo de utilização de medicamentos a partir de prescrições em um hospital do Rio Grande do Sul. Nesse estudo, a dipirona foi o fármaco mais prescrito em ambiente hospitalar (WEBER; BUENO; OLIVEIRA, 2012).

Ressalta-se que a dipirona, além de ser o mais prescrito, foi o fármaco de maior destaque, com provável interação comparado a outros fármacos, em um total de 15 fármacos diferentes (captopril, hidrocortisona, furosemida, levofloxacino, enoxaparina, tenoxicam, dexametasona, amitriptilina, losartana, hidroclorotiazida, prednisolona, enalapril, caverdilol, espironolactona, ácido acetilsalicílico), seguida pela furosemida, com a possível interação com 11 fármacos e o captopril com 10 fármacos diferentes (Gráfico 3). 
Tabela 3. Fármacos mais prescritos no Hospital Municipal de Cuité.

\begin{tabular}{lcc}
\hline \multicolumn{1}{c}{ Fármacos } & Número de prescrições & (\%) \\
\hline Dipirona & 129 & 39,7 \\
Hidrocortisona & 120 & 36,9 \\
Ceftriaxona & 117 & 36,0 \\
Brometo de ipratrópio & 67 & 20,6 \\
Sulfato de salbutamol & 48 & 14,8 \\
Cloridrato de ondansetrona & 46 & 14,2 \\
Butilbrometo de scopolamina & 43 & 13,2 \\
Cefalotina & 35 & 10,8 \\
Furosemida & 26 & 8,0 \\
\hline
\end{tabular}

Em um estudo realizado em Porto Alegre-RS, os fármacos mais envolvidos nas interações foram dipirona sódica, captopril e ácido acetilsalicílico (PIVATTO et al. 2009), resultado semelhante ao encontrado no presente estudo. Nesse contexto, destaca-se a interação entre dipirona e hidrocortisona. Esta associação é considerada como uma interação medicamentosa potencial de severidade maior, pois pode aumentar o risco de úlcera gastrointestinal e sangramento (MICROMEDEX, 2017).

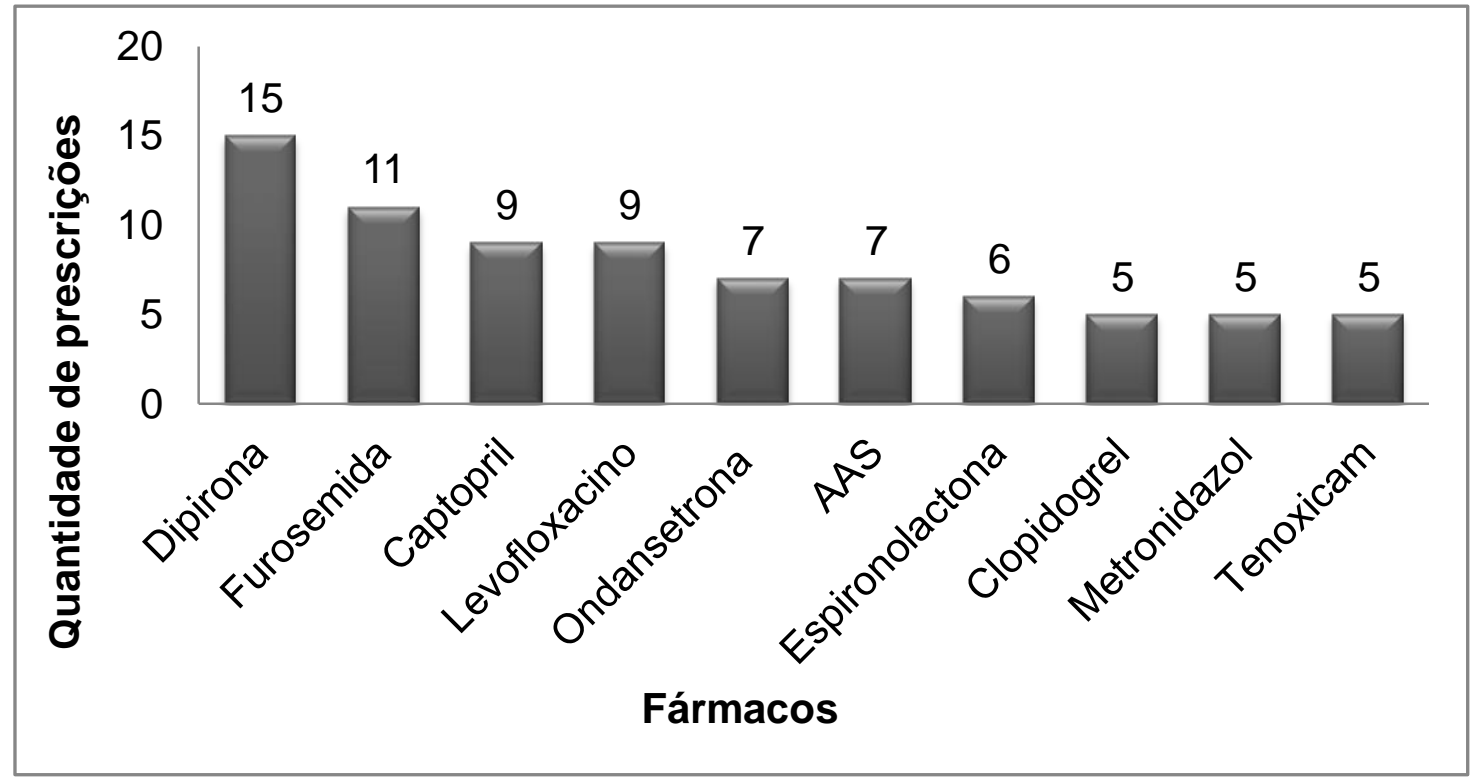

Gráfico 3. Fármacos mais envolvidos em potenciais interações nas prescrições hospitalares

Fonte: elaboração própria. 
Com relação à furosemida e o captopril, esses fármacos podem interagir com vários outros, ou mesmo com alimentos, sendo que algumas interações oferecem riscos potenciais ao paciente, a exemplo da interação da furosemida com a dipirona que pode ocasionar a diminuição de prostaglandinas renais afetando o correto funcionamento dos rins (LIMA et al., 2011).

Observou-se dentre as 120 prescrições as 10 interações medicamentosas mais prevalentes e sua classificação quanto ao mecanismo de ação, severidade, início de ação e documentação (Quadro 1), no qual estas potenciais interações correspondem a $46,7 \%$ de todas as interações encontradas no estudo.

Entre as potenciais interações encontradas, observou-se que, em relação ao grau de severidade, 128 (60\%) foram classificadas como de severidade maior, o que evidencia um alto número de interações com relevada importância clínica. Outras 70 (33\%) potenciais interações medicamentosas, foram evidenciadas com severidade moderada e 1 (0,5\%) foi considerada contraindicada (Gráfico 4).

As potenciais interações encontradas também foram classificadas de acordo com o início de ação em: não especificado, tardio e rápido. Nesse estudo, $69,2 \%$ possuíam um início não especificado, 22,4\% são de início tardio e $8,4 \%$ possuíam início rápido (Gráfico 5 ).

As interações consideradas de severidade maior podem ser definidas como aquelas que oferecem risco de morte e/ou necessitam de intervenção profissional imediata. Um exemplo ocorre com o uso de furosemida e gentamicina que deve ser evitado devido à potencial nefrotoxicidade aditiva $e$ ototoxicidade. A administração simultânea de furosemida intravenosa pode aumentar a toxicidade e alterar as concentrações corporais de gentamicina. Se for necessária uma terapia simultânea, é indispensável o controle da ototoxicidade aditiva e nefrotoxicidade. É relatado que a furosemida pode aumentar a depuração renal da gentamicina durante o período de diurese, bem como, a depuração plasmática média total da gentamicina diminui após a furosemida. São interações de moderada severidade as que podem resultar em exacerbação das condições clínicas e/ou necessitam de mudança na terapia. 
Tem-se como exemplo a interação entre levofloxacino e tenoxicam. A administração simultânea de levofloxacino, um antimicrobiano do tipo de quinolona e um anti-inflamatório não-esteroidal, pode aumentar o risco de estimulação do SNC e causar crises convulsivas (MICROMEDEX, 2017).

Quadro 1. Interações mais prevalentes nas prescrições e sua classificação

\begin{tabular}{|c|c|c|c|c|}
\hline Interação & $\begin{array}{c}\text { Grau de } \\
\text { severidade }\end{array}$ & $\begin{array}{l}\text { Início de } \\
\text { ação }\end{array}$ & $\begin{array}{c}\text { Embasamento } \\
\text { na literatura }\end{array}$ & $\begin{array}{l}\text { Mecanismo de } \\
\text { ação }\end{array}$ \\
\hline $\begin{array}{c}\text { Dipirona + } \\
\text { hidrocortisona }\end{array}$ & Maior & $\begin{array}{c}\text { Não } \\
\text { especificado }\end{array}$ & Razoável & Efeitos aditivos \\
\hline $\begin{array}{c}\text { Dipirona + } \\
\text { levofloxacino }\end{array}$ & Moderado & Tardio & Razoável & $\begin{array}{c}\text { Estimulação do } \\
\text { SNC }\end{array}$ \\
\hline $\begin{array}{c}\text { Dipirona + } \\
\text { ondansetrona }\end{array}$ & Maior & $\begin{array}{c}\text { Não } \\
\text { especificado }\end{array}$ & Bom & $\begin{array}{c}\downarrow \text { Função } \\
\text { plaquetária }\end{array}$ \\
\hline $\begin{array}{l}\text { Dipirona + } \\
\text { furosemida }\end{array}$ & Maior & $\begin{array}{c}\text { Não } \\
\text { especificado }\end{array}$ & Bom & $\begin{array}{c}\downarrow \text { Prostaglandinas } \\
\text { renais }\end{array}$ \\
\hline $\begin{array}{l}\text { Furosemida + } \\
\text { hidrocortisona }\end{array}$ & Moderado & Tardio & Razoável & Efeitos aditivos \\
\hline $\begin{array}{l}\text { Dipirona + } \\
\text { tenoxicam }\end{array}$ & Maior & $\begin{array}{c}\text { Não } \\
\text { especificado }\end{array}$ & Razoável & $\begin{array}{c}\text { Efeitos aditivos na } \\
\text { hemostasia }\end{array}$ \\
\hline $\begin{array}{c}\text { Dipirona + } \\
\text { dexametasona }\end{array}$ & Maior & $\begin{array}{c}\text { Não } \\
\text { especificado }\end{array}$ & Razoável & Efeitos aditivos \\
\hline $\begin{array}{c}\text { Furosemida + } \\
\text { captopril }\end{array}$ & Moderado & Rápido & Bom & $\begin{array}{c}\downarrow \text { Volume } \\
\text { intravascular e } \\
\text { vasodilatação }\end{array}$ \\
\hline $\begin{array}{c}\text { AAS + } \\
\text { clopidogrel }\end{array}$ & Maior & $\begin{array}{c}\text { Não } \\
\text { especificado }\end{array}$ & Razoável & Efeitos aditivos \\
\hline $\begin{array}{c}\text { Ondansetrona } \\
+ \\
\text { Metronidazol }\end{array}$ & Maior & $\begin{array}{c}\text { Não } \\
\text { especificado }\end{array}$ & Razoável & $\begin{array}{c}\text { Efeitos aditivos } \\
\text { sobre o intervalo } \\
\text { QT }\end{array}$ \\
\hline
\end{tabular}

Fonte: elaboração própria. 


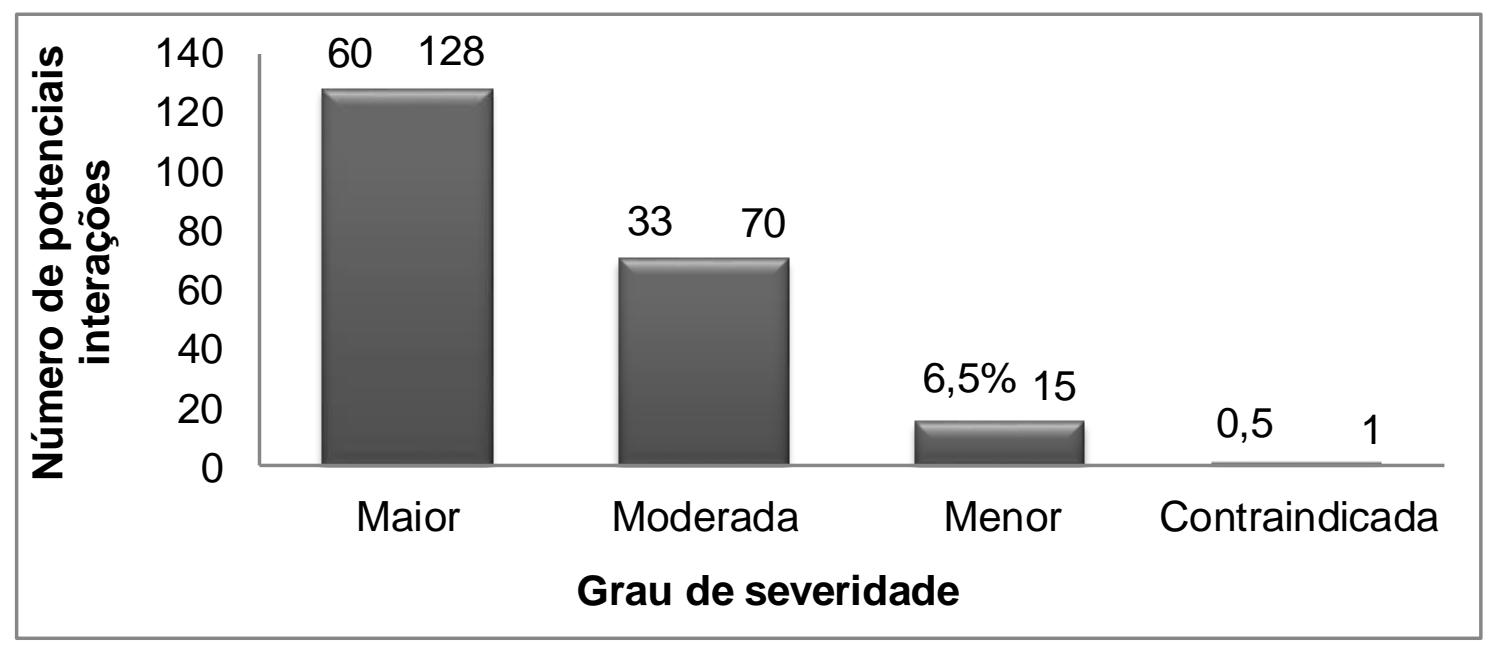

Gráfico 4. Classificação das interações medicamentosas quanto ao início de ação

Fonte: elaboração própria.

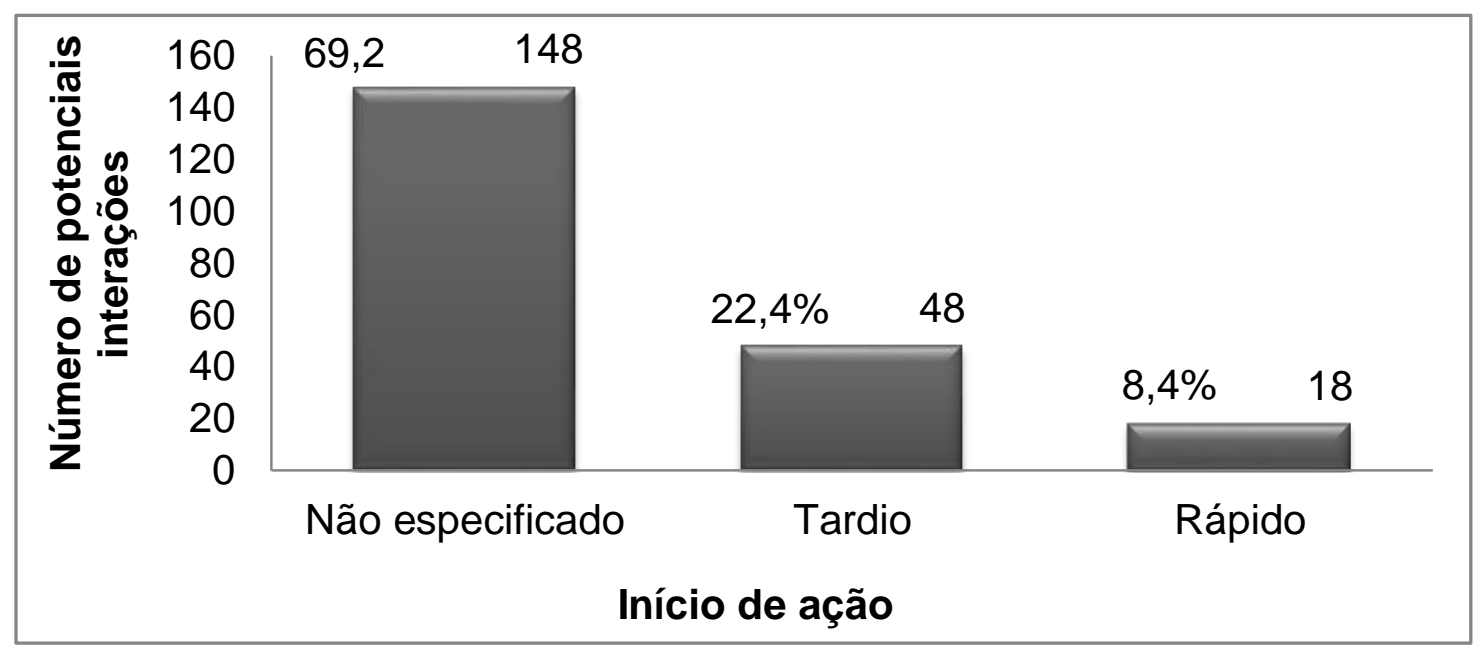

Gráfico 5. Classificação das interações quanto ao grau de severidade da interação medicamentosa

Fonte: elaboração própria.

As interações de severidade menor ocorrem quando o paciente apresenta alterações no quadro clínico, porém sem exigir alterações na terapia medicamentosa. A interação entre ampicilina e gentamicina é um exemplo desse tipo de interação medicamentosa encontrada nesse estudo, podendo ser considerada benéfica. São classificadas como interações contraindicadas aquelas que demandam intervenção imediata, pois representam risco de vida iminente. Um exemplo ocorre com o uso concomitante de cetoconazol com cloridrato de ondansetrona, um substrato CYP3A4 conhecido por prolongar o 
intervalo QT. Essa interação medicamentosa pode gerar um efeito aditivo, e por isso é contraindicada (AFIUNE et al., 2016; MICROMEDEX, 2017).

No tocante à documentação, $32,2 \%$ das interações medicamentosas possuíam um bom embasamento na literatura, 60,4\% uma documentação razoável e 7,4\% eram tidas como excelentes quanto à documentação, não havendo interação com grau de conhecimento pobre ou improvável (Gráfico 6).

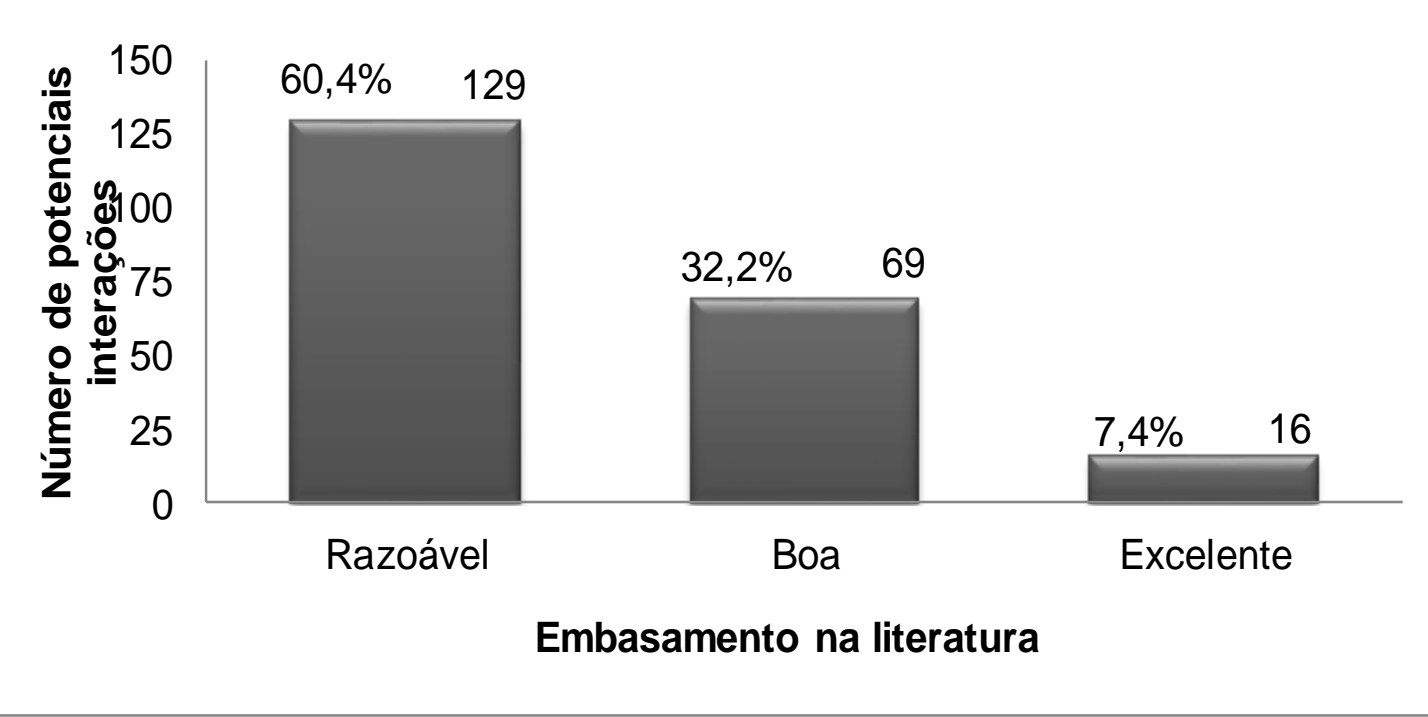

Gráfico 6. Classificação das interações medicamentosas quanto ao embasamento na literatura

Fonte: elaboração própria.

Um exemplo de potencial interação de início não especificado encontrado no presente trabalho ocorre com a associação de furosemida e morfina, sendo esse analgésico um indutor da liberação de hormônio antidiurético, podendo resultar em eficácia reduzida da furosemida. Com relação à interação medicamentosa com início de ação tardio, pode-se citar a que ocorre entre a levotiroxina e o omeprazol, que resulta no aumento dos níveis de hormônio tireoestimulante, no entanto, seu mecanismo de ação é desconhecido. Como exemplo de interação de início rápido, foi observada na associação entre amiodarona e fentanil. A amiodarona inibe o metabolismo do fentanil por meio do CYP4503A4, podendo causar cardiotoxicidade e aumento dos efeitos tóxicos do fentanil, caracterizando-se como uma interação medicamentosa de mecanismo de ação farmacocinético (MICROMEDEX, 2017). 
A documentação científica apresenta-se como excelente quando estudos controlados estabelecem à existência da interação medicamentosa; boa, quando uma documentação forte sugere a existência da interação, mas faltam estudos bem controlados (GOTARDELO et al., 2014). As interações que são de documentação razoável são as que possuem pobre documentação, mas existem considerações farmacológicas para a sua existência (PINTO; SOUZA; CARNEIRO, 2015).

Nem todas as interações são clinicamente relevantes e, mesmo na presença de associações com riscos moderados a graves, antes da substituição ou suspensão dos fármacos envolvidos, admite-se a monitorização clínico-laboratorial e a verificação da relação risco-benefício quanto ao seu uso (GOTARDELO et al., 2014).

Assim, é evidente a importância da inclusão do profissional farmacêutico na equipe multidisciplinar. Este profissional pode colaborar de forma significante na segurança e eficácia da terapia do paciente, avaliando as prescrições e identificando possíveis interações medicamentosas. 0 profissional farmacêutico irá precaver a ocorrência desses acontecimentos e, junto a equipe de saúde, irá avaliar o risco/benefício para decidir sobre a manutenção ou mudança na terapia prescrita, bem como, auxiliar na redução dos riscos derivados da farmacoterapia.

\section{Conclusão}

Pode-se observar a existência de uma alta prevalência de potencias interações medicamentosas nas prescrições do Hospital Municipal de Cuité/PB. As potenciais interações estavam presentes tanto em prescrições para pacientes do sexo masculino, quanto feminino, principalmente em prescrições para idosos. Adicionalmente, quanto maior o número de fármacos por prescrição, maior o número de potenciais interações. Os fármacos que estão entre os mais prescritos, como a dipirona, também estão entre os que mais se envolveram em interações. Em sua maioria, as interações medicamentosas apresentaram um grau de severidade maior, início de ação não especificado e razoável documentação. 
Estas informações demonstradas no estudo, relacionadas também aos tipos de interações e frequência, são de suma importância para os profissionais de saúde, sobretudo os que atuam em hospitais, a fim de reduzir a ocorrência de possíveis interações e tornar imprescindível a monitorização das futuras prescrições. Dessa forma, é possível aperfeiçoar o manejo clínico, evitando complicações farmacológicas e contribuindo para a saúde da população.

\section{Referências}

AFIUNE, L. A. F. et al. Potenciais interações medicamentosas em prescrições oriundas do hospital municipal e pronto socorro de Barra do Garças/MT. Revista Contexto \& Saúde, v. 16, n. 31, p. 128-139, dez. 2016.

CEDRAZ, K. N.; DOS SANTOS JÚNIOR, M. C. Identificação e caracterização de interações medicamentosas em prescrições médicas da unidade de terapia intensiva de um hospital público da cidade de Feira de Santana, BA. Revista da Sociedade Brasileira de Clínica Médica, v. 2, n. 12, p. 1-7, abr-jun. 2014.

COELHO, P. V.; BRUM, C. A. Interações de medicamentos agentes antibacterianos em prescrições de um hospital público de Minas Gerais. Revista Brasileira de Farmácia, v. 93, n. 3, p. 341-346, jul-set. 2012.

COSTA, M. P.; CATARINA, P.; BARATA, P. Avaliação do perfil de prescrição num serviço hospitalar de psiquiatria. Acta Farmacêutica Portuguesa, v. 3, n. 2, p. 105112, jun. 2014.

FERREIRA JR, C. L. et al. Análise das Interações Medicamentosas em Prescrições de uma Instituição de Longa Permanência em um Município de Minas Gerais. Boletim Informativo Geum, v. 7, n. 1, p. 64-70, jan-mar. 2016.

FORMIGA, L. M. F. Interação medicamentosa: conhecimento dos enfermeiros de um hospital público do Piauí. 2012. 74 f. Dissertação (Mestrado em Farmacologia) Programa de Pós-Graduação em Farmacologia, Universidade Federal do Ceará, Fortaleza 2012.

GOTARDELO, D. R. et al. Prevalência e fatores associados a potenciais interações medicamentosas entre idosos em um estudo de base populacional. Revista Brasileira de Medicina de Família e Comunidade, v. 31, n. 9, p. 111-118, abr. 2014.

LIMA, C. L. P. et al. Interações medicamentosas na hipertensão: Papel do farmacêutico no acompanhamento clínico dos pacientes. Cadernos de Graduação de Ciências Biológicas e da Saúde, v. 13, n. 14, p. 69-81, jul-dez. 2011.

LIMA, T. A. M. et al. Analysis of potential drug interactions and adverse reactions to nonsteroidal anti-inflammatory drugs among the elderly. Revista Brasileira de Geriatria e Gerontologia, v. 19, n. 3, p. 533-544, mai-jun. 2016. 
MARSILIO, N. R.; SILVA, D.; BUENO, D. Drug incompatibilities in the adult intensive care unit of a university hospital. Revista Brasileira de Terapia Intensiva, v. 28, n. 2, p. 147-153, apr-jun. 2016.

MICROMEDEX ${ }^{\circledR}$ Healthcare Series. Thomson. Base de Dados. Disponível em: https://www.thomsonhc.com/hcs/librarian/CS/70A4EC/PFActionld/pf.HomePage/ssl/tru e. Acesso em: 19 de ago. 2017.

MOURA, C. S.; TAVARES, L. S.; ACURCIO, F. A. Interação medicamentosa associada à reinternação hospitalar: estudo retrospectivo em um hospital geral. Revista de Saúde Pública, v. 46, n. 6, p. 1082-1091, dez. 2012.

PINTO, L. H.; SOUZA, H.; CARNEIRO, T. K. Avaliação da frequência de interações medicamentosas ocorridas com pacientes internados em clínica cirúrgica em um hospital público de Joinville. Revista Eletrônica de Farmácia, v. 7, n. 2, p. 16-29, jun. 2015.

PINTO, N. B. F. et al. Interações medicamentosas em prescrições de idosos hipertensos: prevalência e significância clínica. Revista de Enfermagem UERJ, v. 22, n. 6, p. 785-791, nov-dez. 2014.

PIVATTO, J. F. et al. Potenciais interações medicamentosas em prescrições de um hospital-escola de Porto Alegre. Revista AMRIGS, v. 3, n. 53, p. 251-256, jul-set. 2009.

QUEIROZ, K. C. B. et al. Análise de interações medicamentosas identificadas em prescrições da UTI neonatal da ICU-HGU. UNOPAR Científica. Ciências Biológicas e da Saúde, v. 3, n. 16, p. 203-207, jul. 2014.

ROCHA, P. C. F.; MOTA, P. S.; OLIVEIRA, C. I. F. B. Prevalência de potenciais interações medicamentosas em uma unidade de terapia intensiva de Manaus-AM. Revista Brasileira de Farmácia, v. 95, n. 3, p. 909-923, set-dez. 2014.

SANTOS, F. S.; FREITAS, P. E.; COSTA, J. M. Associação entre ocorrência de reações adversas e realização de intervenções farmacêuticas em um hospital de ensino. Revista Brasileira de Farmácia Hospitalar e Serviços de Saúde, v. 7, n. 2, p. 8-14, abr-jun. 2016.

SANTOS, H. C. et al. Possíveis interações medicamentosas com psicotrópicos encontradas em pacientes da Zona Leste de São Paulo. Revista de Ciências Farmacêuticas Básica e Aplicada, v. 30, n. 3, p. 285-289, dez. 2010.

SCRIGNOLI, C. P.; TEXEIRA, V. C. M. C.; LEAL, D. C. P. Interações medicamentosas entre fármacos mais prescritos em unidade de terapia intensiva adulta. Revista Brasileira de Farmácia Hospitalar e Serviços de Saúde, v. 7, n. 2, p. 26-30, abr-jun. 2016.

VARALLO, F. R.; COSTA, M. A.; MASTROIANNI, P. C. Potenciais interações medicamentosas responsáveis por internações hospitalares. Revista de Ciências Farmacêuticas Básica e Aplicada, v. 1, n. 34, p. 79-85, jan-abr. 2013.

VIEL, A. M. et al. Interações medicamentosas potenciais com benzodiazepínicos em prescrições médicas de pacientes hospitalizados. Revista de Ciências Farmacêuticas Básica e Aplicada, v. 35, n. 4, p. 589-596, out-dez. 2014. 
WEBER, D.; BUENO, C. S.; OLIVEIRA, K. R. Análise das prescrições medicamentosas de um hospital de pequeno porte do noroeste do Estado do Rio Grande do Sul. Revista de Ciências Farmacêuticas Básica e Aplicada, v. 33, n. 1, p. 139-145, jan-mar. 2012. 